\title{
Les Volcans Eteints Du Maroc: Patrimoine Geologique Encore Peu Connu "Etude De Cas"
}

\author{
Aâtika Eddif, PhD \\ Laboratoire de Recherche Scientifique et Développement Pédagogique \\ Centre Régional des Métiers de l'Education et de la Formation, \\ Meknès, Maroc \\ Hassan Ouazzani, PES \\ Université Moulay Ismail, Faculté des Sciences Meknès, Maroc \\ Driss Sadkaoui, PA \\ Amina Tajd, PA \\ Ahmed Aghbal, PhD \\ Ahmed Hamid, Inspecteur SVT \\ Laboratoire de Recherche Scientifique et Développement Pédagogique \\ Centre Régional des Métiers de l'Education et de la Formation, \\ Meknès, Maroc
}

Doi: 10.19044/esj.2017.v13n33p90 URL:http://dx.doi.org/10.19044/esj.2017.v13n33p90

\begin{abstract}
The present study aims to identify the degree of knowledge with regard to the Moroccan volcanic inheritance known as "geosites". More precisely, it has to do with those of the Middle Atlas with the school students and the future teachers of the Sciences of Life and Earth (SVT). It also involves their reflection on the likely means to make known and to share this geological inheritance with the target population and with the general public. This, however, is aimed at sensitizing its conservation for pedagogical, scientific, and ecotouristic awareness. In order to answer our study's objectives, we administered an anonymous questionnaire to 232 school students in 2nd year of the secondary school of the provincial delegations of education of Meknes and Sale, and to 26 future teachers of the SVT of the regional center for education and training professions in Meknes, Morocco. The results of the research showed that these volcanoes are unknown among respondents with proportions of $80 \%$ among school students and $8 \%$ among future teachers.
\end{abstract}

Keywords: Volcanic, inheritance, geosite, pedagogy, ecotouristic, sensibilize, Science of Life and Earth 


\section{Résumé}

La présente étude se propose d'identifier le degré de connaissance à l'égard du patrimoine volcanique marocain, "géosites" plus précisément, ceux du Moyen Atlas auprès des apprenants et des futurs enseignants des Sciences de la Vie et de Terre (SVT), et la réflexion sur les moyens susceptibles de faire connaître et partager à la population cible et au grand public ce patrimoine géologique afin, de sensibiliser à sa conservation à des fins pédagogiques, scientifiques, écotouristiques,... Pour répondre à nos objectifs, nous avons mené des enquêtes sous forme d'un questionnaire anonyme distribué auprès de 232 apprenants de la 2éme année du cycle secondaire collégial des délégations provinciales de l'éducation de Meknès et de Salé et de 26 futurs enseignants des SVT du centre régional des métiers de l'éducation et de la formation de Meknès, Maroc. Les résultats de la recherche ont montré que ces volcans sont méconnus auprès des interrogés avec des proportions de $80 \%$ chez les apprenants et $8 \%$ chez les futurs enseignants.

Mots-cles: Patrimoine, volcan, géosites, pédagogie, écotourisme, sensibilisation, Science de la Vie et de la Terre.

\section{Introduction}

Le Maroc se situe à la pointe nord-ouest du continent africain. Son géologique se succède depuis le Précambrien jusqu' à l'Alpin. De telle histoire procure une remarquable variété des domaines structuraux. On y distingue en allant du Sud au Nord: le domaine anti-atlasique et son prolongement saharien façonné par les orogenèses précambriennes et varisque. Par contre le domaine atlasique et mésétien et le domaine rifain se caractérisent surtout par l'empreinte des orogenèses varisque et alpine (Piqué et al., 1994; Piqué et al., 2007; Michard, 1976) (Figure 1).

Cette partie nord de l'Afrique (Maroc) a connu plusieurs éruptions volcaniques dont certaines sont soit d'âge précambrien (exemple : le volcan de Jbel Boho; les volcans de Siroua et de Saghro dans l'Anti-Atlas), soit appartiennent au Trias, tandis que d'autres sont d'âge Tertiaire ou Quaternaire (exemple : le volcan de Gourogou dans le domaine rifain, et les volcans de Hebri, Habri, ...ect de la chaîne volcanique dans le domaine du Moyen Atlas) (Figure 2). L'ensemble de ces volcans anciens associé à d'autres sites géologiques de nature et de dimension variable allant de l'échelle microscopique à l'échelle de l'affleurement constitue un patrimoine riche et diversifié méritant d'être mis en valeur et protégé.

Ces géosites à valeur patrimoniale offrent un potentiel élevé pour des usages scientifiques, pédagogique par excellence mais aussi récréatifs, 
scénique, culturel, économique et géotouristique dans le cadre du développement durable.

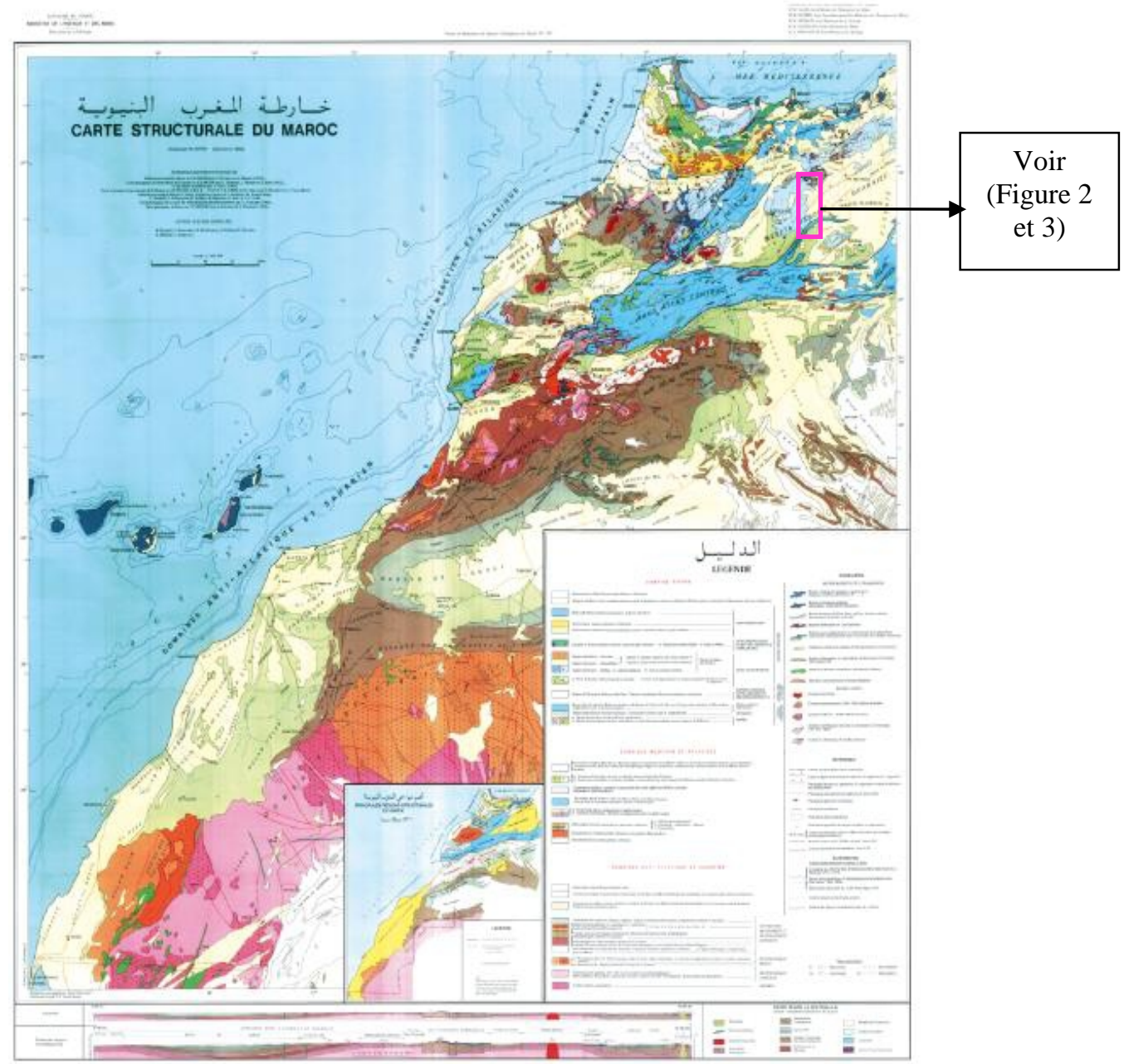

Figure 1. Domaines structuraux du Maroc 


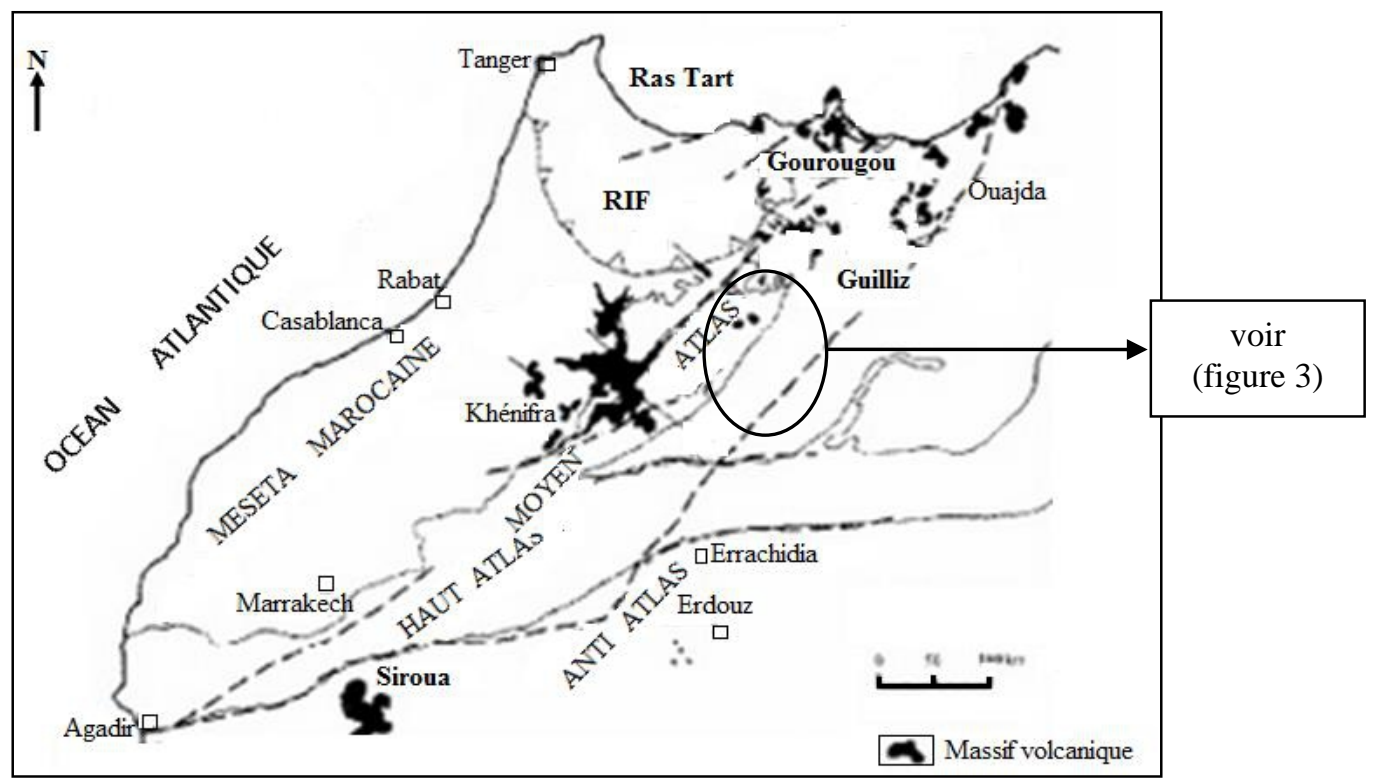

Figure 2. Distribution du volcanisme néogène et quaternaire du Maroc (El Azzouzi et al., 2010)

Qu'entend-on par «patrimoine»? le concept patrimoine est bien intégré dans le vocabulaire commun, il est défini par (UNESCO, 1972) comme étant "l'héritage du passé dont nous profitons aujourd'hui et que nous transmettons aux générations à venir". Plus récemment, ce concept est utilisé d'une façon beaucoup plus holistique pour établir le lien avec d'autres concepts. Ainsi la littérature a permis d'offrir plusieurs concepts entre autres:

Le «patrimoine géologique» qui selon (Jonin, 2013) "a ses racines au sein de la protection de la nature, et en particulier dans les réserves naturelles de France (RNF) depuis (1985).

Pour Billet (2002; cité par De Wever, 2009), le patrimoine géologique désigne « tous les témoins de l'histoire de la Terre qui participent de la connaissance des événements physiques et biologiques qui ont marqué notre planète ». Dans la même optique (BRGM, 2013) le définit comme étant "tous les objets et sites qui symbolisent la mémoire de la Terre, de l'échelle de l'échantillon à l'échelle des paysages."«géosite» peut être réservé à des sites d'intérêt géologique sensu lato à l'échelle kilométrique ou du paysage. On trouve aussi «géodiversité» (Gray, 2001), «géopatrimoine» (Panizza, 2001; Reynard, 2009; Reynard, 2005; Giusti, 2013) et «géomorphosite» ou «patrimoine géomorphologique» est un concept récent qui a été introduit par (Panizza, 2001) et repris par (Pereira, 2005; Reynard, 2008; Sellier, 2009; Portal, 2010; André, 2013) : il désigne l'ensemble des formes du relief -de l'échelle des microformes à celle des reliefs majeurs- qu'une société 
considère comme digne d'être conservé et transmis aux générations futures. (André, 2013; cité par Bétard, 2016).

Dans cette perspective, cette étude s'inscrit dans le cadre du patrimoine géologique marocain relatif aux volcans, particulièrement ceux du Moyen Atlas. L'intérêt que nous portons à ce concept en tant que chercheurs et formateurs des futurs enseignants des SVT découle de deux raisons principales : d'une part, l'existence de profondes lacunes dans les connaissances de la majorité des futurs enseignants des SVT du CRMEF Meknès et de leurs futurs apprenants relatives à l'existence de volcans éteints au Maroc entre autres "la chaîne volcanique du Moyen Atlas (Eddif et al., 2016a; Eddif et al., 2016b) d'autre part, la nécessité de valoriser et de sensibiliser le grand public y compris notre population-cible, à la présence de ces volcans mal connus en vue de leur conservation de toute dégradation. La mise en valeur de ce patrimoine volcanique (multitude de géosites volcaniques les plus remarquables) pourrait participer pleinement au développement local et national.

Trois questions de recherche découlent de cette problématique : Quelles sont les spécificités de la chaîne volcanique moyen atlasique? Quelles conceptions relatives à l'existence de volcans au Maroc ont les futurs enseignants des SVT et les apprenants du cycle secondaire collégial? Comment améliorer la connaissance de ce patrimoine?

Pour répondre à ces questions, ce travail s'articule autour de trois objectifs principaux: 1. Mettre en exergue certaines caractéristiques de la chaîne volcanique du Moyen Atlas, 2. Présenter les conceptions relatives à l'existence de volcans éteints au Maroc à la fois chez les futurs enseignants des SVT et des apprenants du cycle secondaire collégial, 3. Proposer quelques moyens susceptibles de faire connaître et partager au grand public ce géosite afin de sensibiliser à sa conservation à des fins pédagogiques, scientifiques, écotouristiques, ...etc

Il est à savoir qu'au Maroc, les volcans (ou volcanisme) figurent dans les programmes des classes : de 2éme année du cycle secondaire collégial, de 2ème du cycle secondaire qualifiant dans l'unité de la tectonique des plaques et voire aussi à l'Université.

\section{Présentation de la Zone D'étude}

La chaîne volcanique qui nous intéresse dans cette étude se situe dans le domaine du Moyen Atlas. Ce dernier est subdivisé par les auteurs en deux domaines : le Causse moyen atlasique au nord-ouest (NW) et le Moyen Atlas plissé au sud-est (SE) qui sont séparés par l'accident nord moyen atlasique (ANMA) orienté NE-SW. Il s'agit d'un ensemble d'une centaine de volcans dénombrés et cartographiés s'étirant selon une direction méridienne N170 (Martin, 1981). Elle s'étend sur près de $120 \mathrm{~km}$; entre El Hajeb et 
Itzer (Figure 3). Ces volcans qui constituent un véritable laboratoire volcanologique remarquable à ciel ouvert! ont fait l'objet d'études pétrograpphiques, géochimiques, radiochronologiques, et didactiques (Martin, 1981; Moukadiri, 1983; Harmand \& Cantagrel, 1984; Harmand \& Moukadiri, 1986; Rachidi, 1995; Morel \& Bellon, 1996; El Azzab \& Wartiti, 1998; Missenard, 1998; El Azzouzi, 1999; EL Azzouzi et al., 2010; Charrière et al., 2011; El Amrani et al., 2014; El Wartiti et al., 2016; Sadkaoui, 2016; Eddif et al., 2016a; Eddif et al., 2016b).

Comme tout le monde devrait le savoir, les volcans éteints au Maroc sont relativement abondants dans les différents domaines structuraux du Maroc comme signalé ci-dessus (Figure 2), en particulier ceux du Moyen Atlas qui constituent un laboratoire volcanologique naturel à ciel ouvert d'une richesse insoupçonnée et d'une relative proximité en raison des courtes distances à parcourir d'un volcan à l'autre de la chaîne; ce qui offrent des opportunités pour le développement du géotourisme et un véritable terrain d'apprentissage pour les apprenants de tous niveaux de classe.

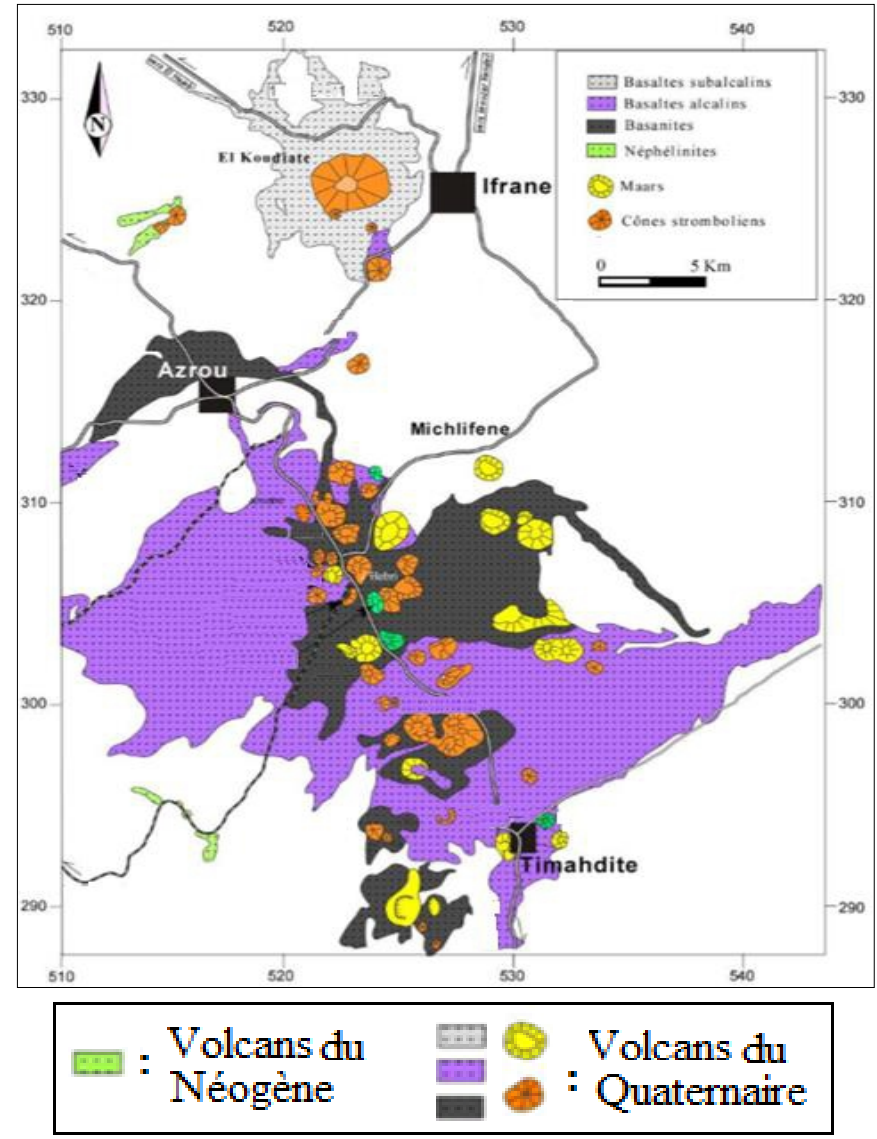

Figure 3. Carte pétrographique des différentes unités volcaniques du Moyen Atlas (EL Azzouzi et al., 2010) 
Dans l'ensemble, l'intérêt géologique de cette chaîne volcanique est basé sur la présence de nombreux volcans associés à des produits volcaniques sous des formes et à des échelles variées (Cendres, bombes, lapilli, ponces...). D'autre part, certains produits volcaniques de types basaltiques sont parsemés d'enclaves de péridotite remontées en surface par le magma lors d'éruption. Ainsi, on distingue plusieurs types d'édifices volcaniques notamment les volcans à cratères ouverts ou égueulés, les volcans à cône simples sans cratère les maars et les édifices composites (Martin, 1981).

Ces différentes caractéristiques géologiques associées à d'autres, font de la chaîne volcanique du Moyen Atlas une multiciplité de géosites "patrimoine géologique" qui mérite d'être reconnu pour qu'il soit protégé.

\section{Matériel et Méthodes}

Plusieurs méthodes ont servi à l'acquisition et à l'exploitation des données. Des sorties géologiques, la collecte des conceptions de la population cible par l'administration d'un questionnaire. Par la suite l'analyse et le traitement des données recueillies au moyen du questionnaire avec Microsoft Office Excel. Des discussions avec les futurs enseignants ont été organisées afin de compléter les informations obtenues à partir du questionnaire de l'enquête.

\section{Présentation de la Population Cible}

La population ciblée par notre étude est constituée de 232 apprenants de la deuxième année du cycle secondaire collégial, répartis sur six classes dans six collèges publics situés dans les délégations provinciales de l'éducation de Meknès et de Salé (Maroc) et de 26 futurs enseignants des SVT au CRMEF, Meknès. Les Tableaux 1 et 2 regroupent quelques caractéristiques de la population cible.

Tableau 1 Quelques caractéristiques de la population cible (apprenants)

\begin{tabular}{|c|c|c|c|c|c|}
\hline population cible & Nombre & âge & niveau d'étude & établissements & villes \\
\hline \multirow[b]{2}{*}{ Apprenants } & \multirow[b]{2}{*}{232} & \multirow{2}{*}{$\begin{array}{c}12 \text { à } \\
14 \text { ans }\end{array}$} & \multirow{2}{*}{$\begin{array}{c}2^{\text {éme }} \text { année du } \\
\text { cycle } \\
\text { secondaire } \\
\text { collégial }\end{array}$} & 3 collèges & Meknès \\
\hline & & & & 3 collèges & Salé \\
\hline
\end{tabular}

Tableau 2 Quelques caractéristiques de la population cible (futurs enseignants

\begin{tabular}{|c|c|c|c|c|c|c|}
\hline $\begin{array}{c}\text { population } \\
\text { cible }\end{array}$ & Nombre & âge & $\begin{array}{l}\text { niveau } \\
\text { d'étude }\end{array}$ & $\begin{array}{l}\text { Options à la } \\
\text { Licence }\end{array}$ & établissements & villes \\
\hline \multirow[t]{2}{*}{$\begin{array}{c}\text { futurs } \\
\text { enseignants }\end{array}$} & \multirow{2}{*}{26} & \multirow{2}{*}{$\begin{array}{c}22 \text { à } \\
30 \text { ans }\end{array}$} & \multirow{2}{*}{$\begin{array}{c}\text { Licence } \\
\text { et } \\
\text { Master }\end{array}$} & $\begin{array}{c}18 \\
\text { biologistes }\end{array}$ & \multirow{2}{*}{$\begin{array}{l}\text { CRMEF } \\
\text { Meknès }\end{array}$} & \multirow{2}{*}{ Meknès } \\
\hline & & & & 8 géologues & & \\
\hline
\end{tabular}




\section{Instrument de Recueil des Données}

Pour la collecte de réponses nous avons eu recours au questionnaire individuel distribué à l'ensemble de la population cible entre 2014 et 2015 avant l'enseignement du concept volcan en classe. Compte tenu de l'arabisation des SVT dans l'enseignement secondaire collégial, le questionnaire mis à la disposition de l'ensemble des enquêtés a été rédigé en langue arabe. Ce questionnaire est composé au total de quatre questions en plus des informations concernant quelques caractéristiques de la population cible, telles que l'âge, le niveau scolaire... (voir tableaux ci-dessus).

les questions sont destinées à recueillir les conceptions relatives de la population cible à définir un volcan par écrit et par dessin, à expliquer comment se forme un volcan, à préciser l'origine du magma des volcans et a exprimer leur accord ou désaccord avec la proposition suivante «y'a-t-il des volcans au Maroc ? » en justifiant leur réponse.

Seules les réponses à cette dernière question seront abordées dans ce qui suit, puisqu'elle est adaptée à la problématique citée ci dessus.

\section{Résultats}

Autour de la question «y'a-t-il des volcans au Maroc ? » justifiez votre réponse. Nous avons fait le décompte des réponses et les avons calculées en pourcentages. Les résultats sont en effet représentés à la fois par un tableau et un camembert ou un graphe.

La plupart des élèves n'ont pas répondu à cette question à environ 80 $\%$ contre $22 \%$ qui ont répondu positivement mais sans pouvoir justifier leurs réponses (Figure 4).

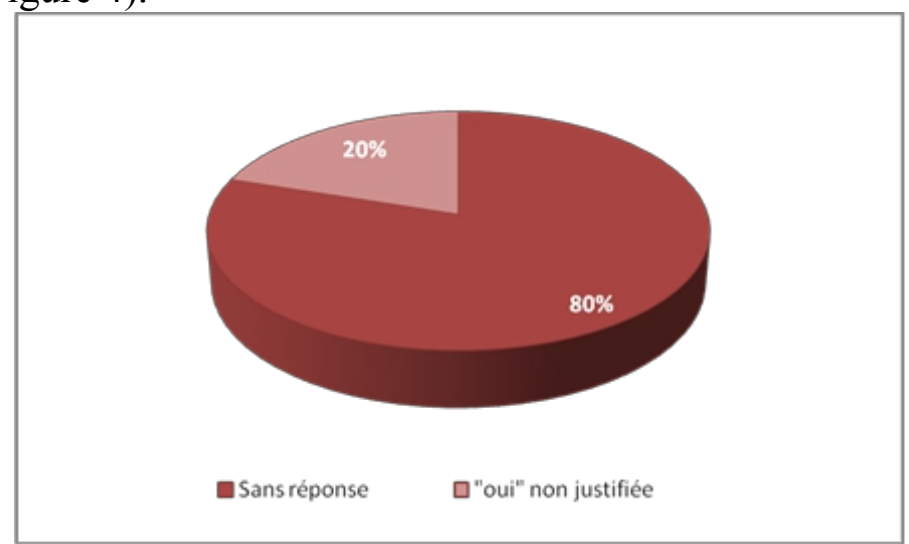

Figure 4. Conceptions des apprenants relatives à l'existence de volcans au Maroc

Du point de vue des enseignants, les résultats collectés sont résumés ci-dessous (Figure 5). Pourcentage des différents types de réponses sur les conceptions relatives à l'existence de volcans éteints au Maroc chez les futurs enseignants d'option biologie et géologie. 


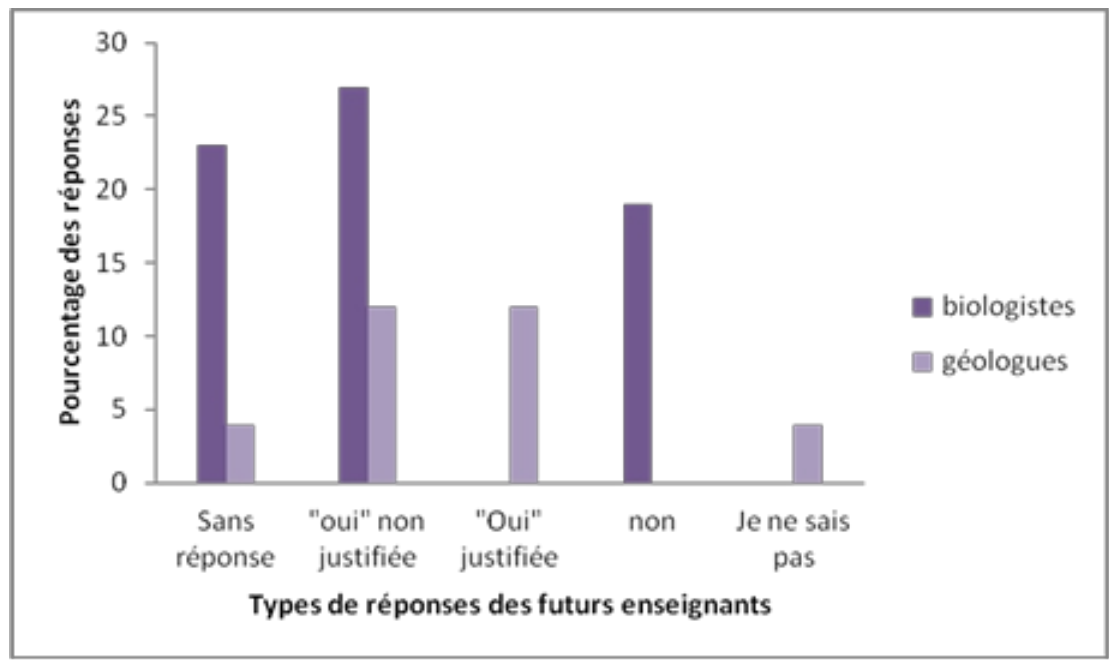

Figure 5. Conceptions des futurs enseignants des SVT relatives à l'existence de volcans au Maroc

En effet, l'étude sur les conceptions relatives à l'existence de volcans au Maroc auprès de nos enquêtés a montré entre autres que : la majorité des futurs enseignants aussi bien les biologistes que les géologues sont inconscients de l'existence de volcans éteints au Maroc entre autres les volcans du Moyen Atlas vu que:

$23 \%$ biologistes contre $4 \%$ géologues n'ont pas donné de réponse, soit $19 \%$ n'ont jamais entendu parler et $4 \%$ géologues ont répondu par "je ne sais pas".

$27 \%$ des enseignants d'option biologie contre $11 \%$ des enseignants d'option géologie ont répondu positivement à la question bien que leur réponse était incomplète puisqu'ils n'ont pas pu la justifier.

Cependant seule $11 \%$ des géologues sont arrivés à répondre juste et ont été en mesure de justifier leurs réponses en donnant l'exemple d'un volcan de la région de Timahdit au Moyen Atlas.

\section{Discussion}

Dans le but d'émerger les conceptions des apprenants du cycle secondaire collégial et des futurs enseignants des SVT, à l'aide d'un questionnaire individuel. L'analyse et le traitement des données collectées attestent que : la plupart des interrogés sont inconscients de l'existence de volcans éteints au Maroc plus particulièrement, ceux du Moyen Atlas. Ces résultats corrèlent avec ceux rapportés dans la littérature (Eddif et al., 2016a; Eddif et al., 2016b).

Il est à noter que seule $20 \%$ des apprenants de notre enquête étaient capables de répondre positivement mais, sans pouvoir justifier leur réponse. Nous ne pouvons, d'un point de vue didactique interpréter ces réponses que 
comme une absence du concept «volcans éteints au Maroc»dans le vocabulaire de ces apprenants de 12-14 ans qui n'avaient étudié auparavant les volcans. Plus encore ces réponses peuvent être liées à des facteurs pédagogiques : d'une part l'absence de ces volcans dans les manuels scolaires en usage au cycle secondaire ; d'autre part, les enseignants exerçants à ce cycle se limitent dans la transposition didactique du concept volcan à des exemples autre que marocains contenues dans de tels manuels.

Quant aux connaissances des futurs enseignants sur les volcans marocains, les résultats ont montré qu'un petit nombre ( 3 futurs enseignants d'option géologie en Licence soit 11\%) est arrivé à répondre. Ceci peut trouver son origine dans des facteurs externes notamment, un parcours universitaire antérieur pluridisciplinaire (Géologie, Biologie Animale ou Végétale, Technologie alimentaire, Ecologie...) dans des universités différentes. Plus encore, d'après l'entretien avec nos enquêtés, il apparaît que les volcans marocains ne sont pas suffisamment approfondie dans l'enseignement universitaire marocain de certaines universités (étude en cours) : En effet, les $11 \%$ cités ci-dessus ont participé à une sortie géologique en lien avec certains volcans du Moyen Atlas (région de Timahdit). Alors que les 5 autres futurs enseignants d'option géologie soit $19 \%$ n'ont jamais entendu parlé de tels volcans. En suite des facteurs plus internes impliquant le manque d'efforts déployés par les enquêtés en recherche et en autoformation.

En outre, ce qui est remarquable dans cet article et dans nos études antérieures (Eddif et al., 2016a; Eddif et al., 2016b) et (en cours) est la stabilité des conceptions relatives à l'existence de volcans éteints au Maroc puisque l'on retrouve à tous niveaux du cursus scolaire ( $\mathrm{du}$ collège à l'université).

\section{Conclusion}

Ce travail présente le résultat d'une étude préliminaire faite sur la chaîne volcanique du moyen atlas qui constitue un patrimoine géologique riche et varié où se concentre une centaine de structures volcaniques variables "géosites" offrant diverses opportunités : pédagogique, scientifique par excellence dont la mesure où ces volcans peuvent constituer un atout permettant aux apprenants de mener en pratique tous les aspects théoriques reçus en classes, touristique, écologique, économique,...etc.

Cependant, ce potentiel patrimonial et insuffisamment connu auprès des apprenants du cycle secondaire et des futurs enseignants des SVT du CRMEF de Meknès. Malheureusement, ce constat est observé aussi bien chez des apprenants à différents niveaux d'enseignement (du collège à l'université) que chez certains enseignants exerçants et par conséquent le niveau scolaire ne se traduit pas par une par une différence dans les 
conceptions relatives à l'existence de volcans éteints au Maroc (étude en cours).

Ceci peut trouver son explication par différents facteurs dits externes et internes. Les facteurs externes sont liés à un défaut de communication entre concepteurs de manuels scolaires et universitaires et par conséquent, cette chaîne volcanique ne figurent dans aucun manuel scolaire, bien que les travaux universitaires sur cette chaîne sont nombreux comme signalé cidessus, d'autres sont dus, est ce que le contenu proposé pour l'enseignement des volcans du Moyen Atlas au niveau des Universités marocaines reste rare et peu traiter. Les facteurs internes sont liés à une transposition didactique au cycle secondaire, du concept volcan concentrée sur le contenu des manuels scolaires, et à un manque d'efforts déployés par les enquêtés en recherche et en autoformation. Pour cela ils restent encore beaucoup d'efforts à faire.

En conséquence, il apparaît nécessaire:

- d'articuler entre les résultats de la recherche des universitaires de différentes disciplines (géologues, géographes, écologistes), des décideurs soucieux de produire des manuels scolaires de qualité ; les enseignants, les didacticiens, ...etc, le tout appuyée par la participation et le soutien de l'état (les Ministères, conseils élus, provinces...) afin d'établir le niveau des intérêts de ce patrimoine géologique à la fois pédagogiques, scientifiques et touristiques...

- d'intégrer la chaîne volcanique du Moyen Atlas dans différents manuels scolaires.

- la nécessité urgente d'instaurer une éducation et un enseignement autour du patrimoine géologique volcanique marocain sous ses volets fondamental et pratique.

- d'agir au niveau de la formation professionnelle des futurs enseignants et de la formation continue des enseignants exerçants des SVT pour leur offrir l'occasion de connaitre les volcans marocains.

En parallèle, il apparaît nécessaire de réaliser de façon urgente plusieurs opérations qui sont possibles et auront des impacts immédiats:

- $\quad$ La nécessité de valoriser, de sensibiliser le grand public y compris les habitants des douars prêt de ces volcans, les apprenants tous cycles confondus, les enseignants, les décideurs de programmes, les responsables locaux et régionaux, collectivités territoriales...à la présence de ces volcans en vue de la conservation de ce patrimoine de toute dégradation et sa participation au développement local et national dans le cadre de la mise en valeur géotouristique de ce patrimoine géologique.

- $\quad$ La nécessité de rendre accessibles ces volcans au plus grand nombre de visiteurs selon leur type (Scientifiques, apprenants tous cycles compris, touristes...) par le biais des aménagements pour la visite, des sentiers, des panneaux indicatifs au niveau des différents voies menant à ces volcans, des 
cartes, des brochures, des expositions, des dépliants publicitaires, des conférences, un site web contenant des cartographies et des fiches sur ces volcans...etc. Avec le besoin d'évaluer les impacts de la fréquentation sur l'ensemble des géosites en tant qu'écosystèmes fragiles.

- $\quad$ Le soutien médiatique par le biais des publications de vulgarisation (presse écrite nationale), des conférences grand public pour que ce patrimoine géologique volcanique puisse franchir la sphère du discours spécialisé et renforcer les connaissances sur ce patrimoine pour le valoriser, le gérer, le préserver et l'aménager durablement et l'évaluer au fil du temps.

\section{References:}

1. André, M.F., Ambert, M., Delannoy, J. J., Hobléa, F., \& Reynard, E. (2013). Géomorphologie et patrimoine. Dans D. Mercier (dir.) Géomorphologie de la France, Dunod, Paris, pp. 201-214.

2. Bétard (2015). Protection et valorisation $d u$ patrimoine géomorphologique en Île-de-France (Bassin de Paris, France). Vol $15, \mathrm{n}^{\circ} 1$.

3. Billet, P. (2002). La protection du patrimoine géologique. Guide juridique, Cahiers techniques de l'ATEN, 76, $148 \mathrm{p}$.

4. Charrière, A., Ouarhache, D., \& El-Arabi, H. (2011). Moyen Atlas. Nouveaux guides géologiques et miniers du Maroc. Notes et Mém, Maroc n559, Vol 4.

5. De Wever, P. (2009). Un inventaire du patrimoine géologique pour la France, La Lettre de 1'OCIM n ${ }^{\circ}$ 121, pp. 12-18.

6. El Amrani, A., \& Furstoss Rodot (2014). Comprendre le volcanisme à travers l'exemple du Moyen-Atlas. Livret de sortie, AEFE - SVT, Zone Maroc SVT 022.

7. El Azzab, D., \& Wartiti, M. (1998). Mise en place de la chaîne volcanique du moyen Atlas (Maroc) : Traitement des données aeromagnetiques. Pangea, $\mathrm{n}^{\circ} 29 / 30$, pp.45-51.

8. EL Azzouzi, M., Maury, R. C., Bellon, H., Youbi, N., Cotton, J., \& Kharbouch, F. (2010). Petrology and K-Ar chronology of the Neogene-Quaternary Middle Atlasbasaltic province. Morocco, Bull. Soc. géol. Fr, t. 181, no 3, pp. 243-257.

9. El Azzouzi, M., Bernard Griffiths, J., Bellon, H., Maury, C. R., Piqué, A., Fourcade, S., Cotton, J. \& Hernandez, J. (1999). Evolution des sources $d u$ volcanisme marocain au cours du Néogène. C.R.Acad. Sci. Paris 329, pp. 95-102.

10. Eddif, A., Selmaoui, S., Abboudi, T., Agorram, B., \& Khzami, S. (2016). Conceptions d'élèves marocains de la deuxième année secondaire collégiale relatives aux volcans. International Journal of Innovation and Scientific Research, Vol. 20 n², pp. 413-427. 
11. Eddif, A., Selmaoui, S., \& Ouazzani, H. (2016). Les volcans : quelles conceptions des futurs enseignants marocains des Sciences de la Vie et de la Terre?. Revue le pédagogue, Vol. 1, n 3/4 pp. 63-77.

12. El Wartiti, M., Malaki, A., El Mahmouhi, N., Azelmad, R., Sadki R., \& Berred, S. (2016). Les géomorphosites du causse moyen atlasique marocain : un circuit géotouristique à développer. International Journal of Innovation and Applied Studies, Vol. 18 no. 3, pp. 773783.

13. Giusti, C. (2013). Les géosites d'intérêt géomorphologique ou géomorphosites dans l'inventaire $d u$ géopatrimoine. Actes $\mathrm{du}$ colloque "Géopatrimoine, un lustre d'inventaire en France ». 10-12 octobre 2012, Digne-les-Bains, Mém. H.S. Soc. Géol. Fr., 13, pp. 2229.

14. Gray (2013). Geodiversity : Valuing and Conserving Abiotic Nature. 2nd Edition. Wiley-Blackwell, Chichester, 508 p.

15. Harmand, C., \& Moukadiri, A. (1986). Synchronisme entre tectonique compressive et volcanisme alcalin: exemple de la province quaternaire du Moyen Atlas (Maroc). B.S.G.F., 8, t.II, pp. 595-603.

16. Harmand, C., \& Cantagrel, J.M. (1984). Le volcanisme alcalin tertiaire et quaternaire du Moyen atlas (Maroc): chronologie K/Ar et cadre géodynamique. J. Afric. EarthSci, vol 2, pp. 51-55.

17. Jonin, M. (2013). Le patrimoine géologique: les mots pour le dire. Communication aux 5èmes journées nationales du patrimoine géologique de Caen 15-18 octobre. https://sgmb.univrennes1.fr/patrimoine-geologique/definition. consulté le 17/08/2016 à 5h. 30

18. Le Tellier, V., Marsteau, C., Regad, J., Carlin, H. Le Goff, E., Audibert, M., Nehlig, P., Rolley J.P., Besset, F., Bakalowicz, M., Crochet, J.Y., et Baillet, L. (2014). Inventaire du patrimoine géologique $d u$ Languedoc-Roussillon. Projets remarquables de BRGM, France. 24p.

19. Martin, J. (1981). Le Moyen Atlas central. Etude géomorphologique. Notes et Mém. Ser. Géol. Maroc n ${ }^{\circ} 258$, et 258 bis, 445p.

20. Michard, A. (1967). Elément de géologie marocaine. Notes et Mém. Serv. Géol. Maroc,

21. Missenard, Y. (2006). Le relief des Atlas Marocains: contribution des processus asthénosphériques et du raccourcissement crustal, aspects chronologiques. Thése d'Etat, Université, Cergy- Pontoise.

22. Morel, J.M., \& Bellon, H. (1996). Le Volcanisme quaternaire du plateau d'Azrou, Maroc. Contribution à la datation isotopique des magmas associés. $13^{\text {ème }}$ Colloque des bassins sédimentaires marocanis, Univ. Cadi Ayyad, Marrakech, 19-22 Mars, p 113. 
23. Moukadiri, A. (1983). Les enclaves ultrabasiques associés aux basaltes alcalins dans le district volcanique d'Azrou Timahdit (Moyen Atlas Maroc). Thèse de 3e cycle. Université de ClemontFerrand II, 150.p.

24. Panizza, M. (2001). Geomorphosites: concepts, methods and example of geomorphological survey. Chinese Science Bulletin, 46, Suppl. Bd, pp. 4-6.

25. Pereira, P., Pereira, D.I., Alves M.I., \& Caetano Brilha J.B. (2005). Geology, landscape and geomorphology: finding the place of geomorphological heritage. IV International Symposium Pro geo on the Conservation of the Geological Heritage, Abstracts. Vol, Braga, University of Minho, Earth Science Centre, p. 10.

26. Piqué, A., Soulaimani, A., Laville, E., Amrhar, M., Bouabdelli, M., Hoepffner, C., \& Chalouan, A. (2007). Géologie du Maroc. Ed Pumag. Marrakech, Maroc, 284 p.

27. Piqué, A. (1994). Géologie du Maroc. Les domaines régionaux et leur évolution structurale. Ed Pumag. Marrakech, Maroc, 284 p.

28. Portal, C. (2010). Reliefs et patrimoine géomorphologique. Applications aux parcs naturels de la façade atlantique européenne. Thèse de doctorat, université de Nantes, $436 \mathrm{p}$.

29. Rachidi, H. N. (1995). Etude du volcanisme plio-quaternaire $d u$ Maroc Central : pétrologie, géochimie et minéralogie. Comparaison avec des laves types du Moyen Atlas et du Rekkam (Maroc). Notes et Mém. Serv. Géol. Maroc, n 381, 157 p.

30. Reynard, E., Coratza, \& Regolini-Bissig (2009). Geomorphosites. Verlag Dr. Friedrich Pfeil, München, 240 p.

31. Reynard, E. (2008). Scientific research and tourist promotion of geomorphological heritage. Geografia Fisicae Dinammica Quaternaria, 31, pp. 225-230.

32. Reynard, E., \& Panizza, M. (2005). Géomorphosites : définition, évaluation et cartographie. Une introduction. Géomorphologie : relief, processus, environnement, 3, pp. 177-180.

33. Sadkaoui, D. (2016). Etude géomorpho-structurale de la région d'Ifrane-Guigou (Moyen Atlas, Maroc). Thèse de Doctorat, Université Sidi Mohamed Ben Abdellah, FST, Fès, 207 p.

34. Sellier, D. (2009). La vulgarisation du patrimoine géomorphologique: objets, moyens et perspectives. Bulletin de l'Association de Géographes Français, 86, 1, pp. 67-81.

35. UNESCO (1972). Convention concernant la protection $d u$ patrimoine mondial culturel et naturel. 17è conférence générale de l'UNESCO. Paris, 16p. 